\title{
Excitation of electrostatic ion-cyclotron-like modes by the electron density ripple in dusty magnetoplasmas
}

\author{
P. K. S H U K L A \\ Institut für Theoretische Physik IV, Ruhr-Universität Bochum, \\ D-44780 Bochum, Germany
}

Also at Department of Physics, Umeå University, SE-90187 Umeå, Sweden; Scottish Universities Physics Alliance (SUPA), Department of Physics, University of Strathelyde, Glasgow G4 ONG, UK; GoLP/Instituto de Plasmas Fusão Nuclear, Instituto Superior Técnico, Universidade Técnica de Lisboa, 1049-001 Lisboa, Portugal; School of Physics, University of KwaZulu-Natal, 4000 Durban, South Africa; Max-Planck Institut für extraterrestrische Physik, D-85740 Garching, Germany

(ps@tp4.rub.de)

(Received 3 March 2009 and accepted 3 March 2009, first published online 18 May 2009)

\begin{abstract}
It is shown that electrostatic ion-cyclotron (EIC)-like modes can be excited by the pre-existing electron density ripple across the external magnetic field in a dusty magnetoplasma. For this purpose, we use the ion continuity and momentum equations, together with the Boltzmann-distributed electrons, and derive the standard Mathieu equation. The latter admits unstable solutions, demonstrating that the EIC-like modes in dusty magnetoplasmas can be driven due to the free energy in the electron density ripple.
\end{abstract}

An electrostatic ion-cyclotron (EIC) wave [1] is supported by inertial magnetized ions and inertialess Boltzmann-distributed electrons. The EIC wave propagates almost perpendicular to the external magnetic field direction, and its frequency is close to the ion gyrofrequency. The EIC wave dispersion comes from the perpendicular ion inertia. The perpendicular (to the external magnetic field direction) phase speed of the EIC wave in a dusty magnetoplasma with immobile negative dust grains is $\left(N_{\mathrm{i} 0} / N_{\mathrm{e} 0}\right)^{1 / 2} C_{\mathrm{s}}[2]$, where $N_{\mathrm{i} 0}=N_{\mathrm{e} 0}+Z_{\mathrm{d}} N_{\mathrm{d} 0}, N_{j 0}$ is the equilibrium number density of the particle species $j$ ( $j$ equals e for electrons, i for ions, and $\mathrm{d}$ for negative dust grains), $Z_{\mathrm{d}}$ is the number of the electrons residing on the dust grains, $C_{\mathrm{s}}=Z_{\mathrm{i}}\left(T_{\mathrm{e}} / m_{\mathrm{i}}\right)^{1 / 2}$ is the ion sound speed, $Z_{\mathrm{i}}$ is the ion charge state, $T_{\mathrm{e}}$ is the electron temperature in energy units, and $m_{\mathrm{i}}$ is the ion mass. The EIC wave in low-temperature laboratory dusty magnetoplasmas has been excited $[3,4]$ by the magnetic field-aligned electron current [5]. It is observed that a substantial amount of negative dust grains makes the electron current driven EIC modes more profound for lower values of the equilibrium electron streaming speed. A comprehensive account of numerous wave phenomena in weakly and strongly coupled dusty plasmas appears in Refs. [6,7]. 
In this letter, we show that the pre-existing electron density ripple across the magnetic field direction can excite EIC-like modes in a magnetized dusty plasma. Physically, the excitation of the EIC-like modes is attributed to the parametric interactions [8] in which a positive energy electron density ripple interacts with the EIC-like modes to produce the EIC-like sidebands, which are negative-energy modes. The energy exchange among the positive- and negative-energy modes leads to the growth of the EIC-like modes, while conserving energy.

Let us consider a dusty magnetoplasma in the presence of inertialess hot electrons, magnetized cold ions, and immobile negative dust grains. The external magnetic field is $\hat{\mathbf{z}} B_{0}$, where $\hat{\mathbf{z}}$ is the unit vector along the $z$ axis in a Cartesian coordinate system, and $B_{0}$ is the strength of the magnetic field. The equilibrium electron number density in the presence of the pre-existing electron density ripple is

$$
n_{\mathrm{e} 0}=N_{\mathrm{e} 0}\left[1+\epsilon \cos \left(k_{\mathrm{e}} x\right)\right]
$$

where $\epsilon$ is the magnitude of the relative (with respect to the equilibrium electron number density $N_{\mathrm{e} 0}$ ) electron density fluctuation, and $k_{\mathrm{e}}$ is the wave number of the electron density ripple.

Since the parallel (to $\hat{\mathbf{z}}$ ) phase speed of the EIC-like modes is much smaller than the electron thermal speed, we obtain from the balance of the parallel (to $\hat{\mathbf{z}}$ ) electric force and the parallel electron pressure gradient the Boltzmann law for the electron number density perturbation

$$
n_{\mathrm{e} 1} \approx n_{\mathrm{e} 0} \frac{e \phi}{T_{\mathrm{e}}}
$$

where $e$ is the magnitude of the electron charge and $\phi$ is the EIC wave potential.

The ion density perturbation $n_{\mathrm{il}}$ associated with the EIC modes is governed by the ion continuity equation

$$
\frac{\partial n_{\mathrm{il}}}{\partial t}+N_{\mathrm{i} 0} \nabla \cdot \mathbf{u}_{\mathrm{i}}=0
$$

where the ion fluid velocity $\mathbf{u}_{\mathrm{i}}$ is determined from the ion momentum equation

$$
\frac{\partial \mathbf{u}_{\mathrm{i}}}{\partial t}+\frac{Z_{\mathrm{i}} e}{m_{\mathrm{i}}}\left(\nabla \phi-\frac{B_{0}}{c} \mathbf{u}_{\mathrm{i}} \times \hat{\mathbf{z}}\right)=0 .
$$

Here $c$ is the speed of light in vacuum. The ions are assumed cold, which is a valid approximation for the EIC wave whose phase velocity is much larger than the ion thermal speed.

Considering the propagation of the EIC-like modes along the $x$ axis, we obtain from (4)

$$
\left(\frac{\partial^{2}}{\partial t^{2}}+\omega_{\mathrm{ci}}^{2}\right) u_{x}+\frac{Z_{\mathrm{i}} e}{m_{\mathrm{i}}} \frac{\partial^{2} \phi}{\partial x \partial t}=0,
$$

where $u_{x}$ is the $x$ component of the ion fluid velocity vector $\mathbf{u}_{\mathrm{i}}$, and $\omega_{\mathrm{ci}}=Z_{\mathrm{i}} e B_{0} / m_{\mathrm{ic}}$ is the ion gyrofrequency.

Eliminating $u_{x}$ from (3) by using (5) with (3), we obtain

$$
\left(\frac{\partial^{2}}{\partial t^{2}}+\omega_{\mathrm{ci}}^{2}\right) n_{\mathrm{i} 1}-\frac{Z_{\mathrm{i}} N_{\mathrm{i} 0} e}{m_{\mathrm{i}}} \frac{\partial^{2} \phi}{\partial x \partial t}=0 .
$$

Finally, replacing $n_{\mathrm{il}}$ in (6) by $n_{\mathrm{el}} / Z_{\mathrm{i}}$ in the quasi-neutrality approximation, which holds for the long-wavelength (in comparison with the electron Debye radius) 
EIC perturbation, we obtain the EIC wave equation

$$
\left(\frac{\partial^{2}}{\partial t^{2}}+\omega_{\mathrm{ci}}^{2}\right)\left[1+\epsilon \cos \left(k_{\mathrm{e}} x\right)\right] \phi-C_{\mathrm{s}}^{2} \frac{\partial^{2} \phi}{\partial x^{2}}=0,
$$

where (2) has been used.

Assuming that the wave potential $\phi$ is proportional to $\exp (-i \omega t)$, where $\omega$ is the frequency, we Fourier transform (7) to obtain

$$
\frac{\partial^{2} \phi}{\partial x^{2}}+\frac{\omega^{2}-\omega_{\mathrm{ci}}^{2}}{C_{\mathrm{s}}^{2}}\left[1+\epsilon \cos \left(k_{\mathrm{e}} x\right)\right] \phi=0 .
$$

Defining $2 \eta=k_{\mathrm{e}} x-\pi$, we express (8) in the standard form of the Mathieu equation

$$
\frac{\partial^{2} \phi}{\partial \eta^{2}}+\frac{4\left(\omega^{2}-\omega_{\mathrm{ci}}^{2}\right)}{k_{\mathrm{e}}^{2} C_{\mathrm{s}}^{2}}[1-\epsilon \cos (2 \eta)] \phi=0 .
$$

Equation (9) admits an instability [9] for $\epsilon_{\mathrm{e}} \ll 1$ and

$$
\frac{2\left(\omega^{2}-\omega_{\mathrm{ci}}^{2}\right)^{1 / 2}}{k_{\mathrm{e}} C_{\mathrm{s}}}=p,
$$

where $p$ is an integer. The instability is strongest for $p \approx 1$, and in that region the growth rate is

$$
\gamma=\frac{p k_{\mathrm{e}} C_{\mathrm{s}}}{4}\left[\frac{\epsilon^{2}}{4}-(p-1)^{2}\right]^{1 / 2} .
$$

For larger $\epsilon$ one can have a strong off-resonant growth rate. For $p \approx 2,3$, etc., the growth rates are smaller; the maximum growth rates for $\epsilon \ll 1$ are $\gamma \approx k_{\mathrm{e}} C_{\mathrm{s}} \epsilon^{2} / 8$ for $p=2$ and $\gamma=81 k_{\mathrm{e}} C_{\mathrm{s}} \epsilon^{3} / 1024$ for $p=3$.

To summarize, we have shown that the pre-existing electron density ripple across the external magnetic field direction can excite the EIC-like modes in a dusty magnetoplasma. The energy stored in the electron density ripple is transferred to the EIC-like modes due to the parametric interactions. As a result, the EIClike modes with frequencies $\left(\omega_{\mathrm{ci}}^{2}+p^{2} k_{\mathrm{e}}^{2} C_{\mathrm{s}}^{2} / 4\right)^{1 / 2}$ grow. The increment is strongest for $p \approx 1$, and it strongly depends on $\epsilon$ and $C_{\mathrm{s}}$. We are hoping that the present theoretical prediction would be verified by forthcoming laboratory experiments.

\section{Acknowledgement}

The author thanks Robert Merlino for enlightening discussions.

\section{References}

[1] Chen, F. F. 1984 Introduction to Plasma Physics and Controlled Fusion, Vol. 1. Plasma Physics. New York: Plenum, pp. 109-112.

[2] Shukla, P. K. and Silin, V. P. 1992 Phys. Scr. 45, 508.

[3] Barkan, A., D’Angelo, N. and Merlino, R. L. 1995 Planet. Space Sci. 43, 905.

[4] Merlino, R. L., Barkan, A., Thompson, C. and D'Angelo, N. 1997 Plasma Phys. Control. Fusion 39, A421. 
[5] Cho, V. and Rosenberg, M. 1995 Planet. Space Sci. 43, 613.

[6] Shukla, P. K. and Mamun, A. A. 2002 Introduction to Dusty Plasma Physics. Bristol: Institute of Physies.

[7] Shukla, P. K. and Eliasson, B. 2009 Rev. Mod. Phys. 81, 25.

[8] Lin, A. T., Kaw, P. K. and Dawson, J. M. 1978 Phys. Rev. A 8, 2618.

[9] McLachlan, N. W. 1964 Theory and Application of Mathieu Functions. New York: Dover, Chapter IV. 